BAPIOAC19-04 


\title{
Lead The Way - Why Emotional Intelligence is Needed for Leaders in 21st Century Primary Care.
}

\author{
Parisah Hussain (presenter for oral presentation), Abdulwahab Ikar, Terrell Okhiria, Aran Sivapalan, \\ Sohini Thakor, Aisha Chaudry, Simran Halari \& Edgar Meyer.
}

Imperial College, London, UK

correspondence toph918@ic.ac.uk

Cite as; Hussain,P, Ikar AW, Okhira T, Sivapalan A, Thakor S, Chaudry A, Halari S \& Meyer E. (2020) Lead The Way. Why Emotional Intelligence is Needed for Leaders in 21st Century Primary Care. The Physician 6(1)c4 DOI; 10.38192/1.6.1.c4

\section{Background}

The need for leadership within 21st Century healthcare is ubiquitous, with better leadership being shown to improve health outcomes. Whether that be leading a ward round, an MDT meeting, or even a cardiopulmonary resuscitation; leadership is a vital necessity in a healthcare setting. When it comes to Primary Care, there is currently a huge demand from patients, colleagues and the NHS, that GPs must meet. With over $50 \%$ of medical students in the UK progressing to specialise in Primary Care, developing leadership skills within this field is vital. Emotional intelligence (EQ) is a core skill found to combine over 12 competencies of leadership. However, soft skills such as EQ have been overlooked during medical training. Our research explores the impact and importance of EQ for leaders in Primary Care.

\section{Methods}

A systematic review highlighted the importance of EQ for healthcare leaders and assesses its impact on key performance indicators. Appropriate search terms were used to systematically search five databases for literature published after 2014. Inclusion and exclusion criteria were applied to screen abstracts and full texts. Final articles included a focus on EQ within healthcare leaders with impact on at least one performance indicator. Primary data collection with GPs was conducted in the form of semi-structured interviews (SSIs) in order to understand the impact of EQ on workplace behaviours and attitudes within Primary Care. Purposive sampling was used to select potential study participants with the aim of providing the most credible information to the study. GPs were recruited through the Royal College of General Practitioners (RCGP) and the Faculty of Medical Leadership and Management (FMLM) and inclusion and exclusion criteria were used to screen participants. 
Ethical approval was received from Imperial College Research Ethics Committee. Interviews were then transcribed, and thematic analysis was used to generate codes and overall themes. Thematic analysis was conducted by analysing each written transcript, generating initial codes, searching for themes and categorising them into overall sub-themes and higher themes.

\section{Results}

The search produced 3638 articles of which 44 were included for full text screening. We found that EQ in healthcare leaders had an impact upon key performance indicators: Job Satisfaction, Patient Satisfaction, Stress, Worker Burnout. A total of 15 SSIs were conducted with GPs across London. From the thematic analysis, 3 higher themes were generated (Patients, Primary Care as an Organisation, and General Practitioners) and 10 sub-themes which portrayed that EQ has a positive impact on job satisfaction, interpersonal relationships, teamwork and levels of burnout for GPs. In addition, EQ was described as being beneficial for patient consultations and thus improving patient satisfaction. With regard to leadership in Primary Care, EQ was found to play a positive role towards change in organisational culture.

\section{Conclusions}

EQ is a widely endorsed skill required for leaders within Primary Care, shown to play an important role for 21st century healthcare. Research determining the efficacy of EQ and its impact in Primary Care can simmer the dispute regarding the importance of EQ within healthcare and ultimately, provide an evidence base to support the creation of EQ training schemes within Primary Care. 